PROCEEDINGS OF THE

AMERICAN MATHEMATICAL SOCIETY

Volume 140, Number 5, May 2012, Pages 1729-1738

S 0002-9939(2011)11091-8

Article electronically published on September 9, 2011

\title{
CR TRANSVERSALITY OF HOLOMORPHIC MAPPINGS BETWEEN GENERIC SUBMANIFOLDS IN COMPLEX SPACES
}

\author{
PETER EBENFELT AND DUONG NGOC SON
}

(Communicated by Franc Forstneric)

\begin{abstract}
We show that a holomorphic mapping sending one generic submanifold into another of the same dimension is CR transversal to the target submanifold provided that the source manifold is of finite type and the map is of generic full rank. This result and its corollaries completely resolve two questions posed by Linda P. Rothschild and the first author in 2006. Another one of our main results, in the special setting of hypersurfaces, was also explicitly stated as a conjecture by B. Lamel and N. Mir in a paper from 2006.
\end{abstract}

\section{INTRODUCTION}

In this paper, we study $\mathrm{CR}$ transversality of holomorphic mappings sending a generic submanifold in $\mathbb{C}^{N}$ into another of the same dimension. Recall that if $U$ is an open subset of $\mathbb{C}^{N}, H$ a holomorphic mapping $U \rightarrow \mathbb{C}^{N}$, and $M^{\prime}$ a generic submanifold through a point $p^{\prime}:=H(p)$ for some $p \in U$, then $H$ is said to be $C R$ transversal to $M^{\prime}$ at $p$ if

$$
T_{p^{\prime}}^{1,0} M^{\prime}+d H\left(T_{p}^{1,0} \mathbb{C}^{N}\right)=T_{p^{\prime}}^{1,0} \mathbb{C}^{N}
$$

where $T^{0,1} M^{\prime}=\mathbb{C} T M^{\prime} \cap T^{0,1} \mathbb{C}^{N}$ denotes the CR bundle on $M^{\prime}$ and $T^{1,0} M^{\prime}=$ $\overline{T^{0,1} M^{\prime}}$ its complex conjugate. We remark that $\mathrm{CR}$ transversality of a holomorphic mapping is a property that in general is strictly stronger than that of transversality as a smooth mapping; i.e., CR transversality implies transversality, but the converse does not hold in general. Transversality is an important and basic notion in geometry and analysis. For instance, an example of a transversality result in analysis is the classical Hopf Lemma for subharmonic functions in a smoothly bounded domain in $\mathbb{R}^{n}$ (see e.g. GT83]). The reader is referred to e.g. the paper [ER06] for further elaboration on the significance of tranversality in geometry and analysis. Also, the reader is referred to Section 2 for definitions and further explanation of the notions that appear in this introductory section.

The problem of $\mathrm{CR}$ transversality has been considered in various situations by many authors; see e.g. Forn76, Forn78, P77, BB82, BR90, BR93, CR94, CR98, BHR95, HP96, ER06, [LM06, BER07 and the references therein. In this paper, we will consider the situation in which there is a generic submanifold

Received by the editors September 25, 2010 and, in revised form, January 25, 2011.

2010 Mathematics Subject Classification. Primary 32H02, 32V20, 32V40.

The first author was partly supported by NSF grant DMS-0701121.

The second author acknowledges a scholarship from the Vietnam Education Foundation for his graduate study at UC San Diego and a grant from NAFOSTED (Vietnam).

(C)2011 American Mathematical Society 1729

Reverts to public domain 28 years from publication 
$M \subset U$, of the same dimension as $M^{\prime}$, such that $p \in M$ and $H(M) \subset M^{\prime}$. In ER06, this same situation is considered and it is proved that the map $H$ is CR transversal to $M^{\prime}$ at $p \in M$ provided that $M^{\prime}$ is of finite type (in the sense of Kohn and Bloom-Graham) at $p^{\prime}:=H(p)$ and the restriction of $H$ to the Segre variety of $M$ at $p$ is finite (as a mapping into the Segre variety of $M^{\prime}$ at $p^{\prime}$ ). The condition on $H$ is satisfied if, for instance, $H$ itself is a finite mapping $\left(\mathbb{C}^{N}, p\right) \rightarrow\left(\mathbb{C}^{N}, p^{\prime}\right)$ (i.e. the inverse image $H^{-1}\left(p^{\prime}\right)$ equals the singleton $\{p\}$ as a germ of a variety at $p$ ). By combining several results including the one mentioned above, it is noted in [ER06] that if $M$ and $M^{\prime}$ are hypersurfaces (i.e. submanifolds of real codimension one) and $M$ is essentially finite at $p$, a stronger condition than finite type in the hypersurface case, then $H$ is $\mathrm{CR}$ transversal to $M^{\prime}$ at $p^{\prime}$ under the assumption that Jac $H \not \equiv 0$, where Jac $H$ denotes the Jacobian determinant of $H$ and $\not \equiv$ means "not identically zero"; we remark that Jac $H \not \equiv 0$ is strictly weaker than being a finite map. It was conjectured in [ER06] that the condition of essential finiteness of $M$ at $p$ could be weakened to finite type at $p$ while maintaining the weak requirement Jac $H \not \equiv 0$ for the mapping. It was also conjectured that the condition Jac $H \not \equiv 0$ would be sufficient to conclude CR tranversality in the case of higher codimensional generic submanifolds $M$ and $M^{\prime}$ provided that $M$ is assumed to be of finite type and essentially finite at $p$. (For higher codimensional generic submanifolds, the conditions of being of finite type and essentially finite are unrelated, and the authors of [ER06] imposed both conditions on $M$ in this conjecture to be on the safe side.) In this paper, we prove both of these conjectures. Indeed, we even prove that the condition of essential finiteness in the higher codimensional case is superfluous. More precisely, we have the following theorem.

Theorem 1.1. Let $M, M^{\prime} \subset \mathbb{C}^{N}$ be smooth generic submanifolds of the same dimension through $p$ and $p^{\prime}$ respectively, and let $H:\left(\mathbb{C}^{N}, p\right) \rightarrow\left(\mathbb{C}^{N}, p^{\prime}\right)$ be a germ of a holomorphic mapping such that $H(M) \subset M^{\prime}$. Assume that $M$ is of finite type at $p$ and $\mathrm{Jac} H \not \equiv 0$. Then $H$ is $C R$ transversal to $M^{\prime}$ at $p^{\prime}$.

We note that if $M^{\prime}$ is of finite type at $p^{\prime}$ and the restriction of $H$ to the Segre variety of $M$ at $p$ is finite, then $M$ is of finite type at $p$ and $\operatorname{Jac} H \not \equiv 0$ (see Proposition 2.3 in [ER06]). Thus, Theorem 1.1] also implies the result in [ER06] described above (Theorem 1.4 in [ER06]).

The condition that $M$ is of finite type at $p$ in Theorem 1.1 is optimal, as the following example shows that being of finite type at most points is not enough.

Example 1.2. Let $H:\left(\mathbb{C}^{2}, 0\right) \rightarrow\left(\mathbb{C}^{2}, 0\right)$ be the mapping $H(z, w)=\left(z, w^{2}\right)$, and $M \subset \mathbb{C}^{2}$ the hypersurface given by $\operatorname{Im} w=(\operatorname{Re} w) \varphi(z, \bar{z})$, where $\varphi$ is a smooth real function such that $\varphi(z, 0) \equiv \varphi(0, \bar{z}) \equiv 0$. Observe that $M$ is parametrized by $(z, \bar{z}, s) \mapsto(z, s+i s \varphi(z, \bar{z}))$. Furthermore

$$
H(z, s+i s \varphi(z, \bar{z}))=\left(z, s^{2}\left(1-\varphi^{2}(z, \bar{z})\right)+2 i s^{2} \varphi(z, \bar{z})\right) .
$$

Then it is easy to see that $H$ sends $M$ into the smooth real hypersurface $M^{\prime}$ given by

$$
\operatorname{Im} w^{\prime}=\frac{2\left(\operatorname{Re} w^{\prime}\right) \varphi\left(z^{\prime}, \bar{z}^{\prime}\right)}{1-\varphi^{2}\left(z^{\prime}, \bar{z}^{\prime}\right)}
$$

Observe that $M$ is of infinite type at 0 since it contains the complex hypersurface $\{w=0\}$; however, $\varphi$ can be chosen such that $M$ is of finite type at most points except for proper subvarieties containing 0 . The reader can check that the map $H$ satisfies Jac $H \not \equiv$ but is not transversal to $M^{\prime}$ at 0 . 
It is also clear that some a priori condition on the mapping $H$ is necessary for the conclusion of Theorem 1.1 to hold, as is easily verified by simple examples. The reader is referred to Example 2.4 in [BER07] (in which one may consider $M \subset \mathbb{C}^{2}$ as a finite type hypersurface in $\mathbb{C}^{3}$ via a standard embedding) for an example of a mapping $H$ (with Jac $H \equiv 0$ ) that is not $\mathrm{CR}$ transversal to $M^{\prime}$ at 0 . If the source manifold $M$ is holomorphically nondegenerate, then in fact the condition Jac $H \not \equiv 0$ is necessary for CR transversality to hold (see Proposition 3.6 below). We state here the following result, which is a direct consequence of Theorem 1.1 and Proposition [3.6. we remark that this result was conjectured in the hypersurfaces case (Conjecture 2.7) in [LM06].

Theorem 1.3. Let $M, M^{\prime} \subset \mathbb{C}^{N}$ be smooth generic submanifolds of the same dimension through $p$ and $p^{\prime}$ respectively, and let $H:\left(\mathbb{C}^{N}, p\right) \rightarrow\left(\mathbb{C}^{N}, p^{\prime}\right)$ be a germ of a holomorphic mapping such that $H(M) \subset M^{\prime}$. Assume that $M$ is holomorphically nondegenerate and of finite type at $p$. Then $H$ is CR transversal to $M^{\prime}$ at $p$ if and only if Jac $H \not \equiv 0$.

If we impose stronger conditions on $M$, such as essential finiteness or finite nondegeneracy in addition to finite type, then we also obtain stronger conclusions as in [ER06]. Indeed, by combining our Theorem 1.1 with Theorems 6.1 and 6.6 in ER06], we obtain the following result; the notions of essential finiteness and finite nondegeneracy will not play a role in any of the proofs in this paper, and, hence, the reader is referred to [ER06] or the book BER99a] for their definitions.

Theorem 1.4. Let $M, M^{\prime} \subset \mathbb{C}^{N}$ be smooth generic submanifolds through $p$ and $p^{\prime}$ respectively, and let $H:\left(\mathbb{C}^{N}, p\right) \rightarrow\left(\mathbb{C}^{N}, p^{\prime}\right)$ be a germ of a holomorphic mapping such that $H(M) \subset M^{\prime}$ and $\operatorname{Jac} H \not \equiv 0$. If $M$ is of finite type and essentially finite at $p$, then $H$ is a finite map $\left(\mathbb{C}^{N}, p\right) \rightarrow\left(\mathbb{C}^{N}, p^{\prime}\right)$. If, in addition, $M$ is finitely nondegenerate at $p$, then $H$ is a local biholomorphism near $p$.

We mention that the notion of CR transversality can be defined also for CR mappings $f: M \rightarrow M^{\prime}$ in terms of a formal power series expansion for $f$ (see e.g. [ER06]). The analog of Theorem 1.1 holds for CR mappings whose differentials have generic maximal rank.

In the next section, we will briefly recall some basic definitions and facts. In Section 3, we will present the proof of Theorem 1.1. For further comments, examples and related results regarding this problem, we refer the reader to e.g. the papers BR90] and ER06.

\section{Preliminaries}

In this section, we will recall some basic definitions and facts about real submanifolds in complex spaces. For more details and proofs of facts stated, we refer the reader to the book BER99a. Recall that a real submanifold $M$ of codimension $d$ in $\mathbb{C}^{N}\left(\cong \mathbb{R}^{2 N}\right)$ is said to be generic if, for every $p \in M$, the submanifold $M$ is defined locally near $p$ by a defining equation $\rho(Z, \bar{Z})=0$, where $\rho=\left(\rho_{1}, \ldots \rho_{d}\right)$ is a smooth $\mathbb{R}^{d}$-valued function satisfying the following condition:

$$
\partial \rho_{1} \wedge \ldots \wedge \partial \rho_{d} \neq 0 \text {. }
$$

In particular, a generic submanifold $M$ of codimension $d$ in $\mathbb{C}^{N}$ is a $\mathrm{CR}$ manifold of CR dimension $n=N-d$. If $M$ is real-analytic and $p \in M$, then there are 
normal (local) coordinates $Z=(z, w)$, where $z=\left(z_{1}, \ldots, z_{n}\right)$ and $w=\left(w_{1}, \ldots, w_{d}\right)$, vanishing at $p$ such that $M$ is defined by the complex equation

$$
w=Q(z, \bar{z}, \bar{w}),
$$

where $Q(z, \chi, \tau)$ is a $\mathbb{C}^{d}$-valued holomorphic function defined in a neighborhood of $p=(0,0)$ satisfying

$$
Q(z, 0, \tau) \equiv Q(0, \chi, \tau) \equiv \tau .
$$

The fact that the $d$ complex equations in (2) define a submanifold of real codimension $d$ is equivalent to the identity

$$
Q(z, \chi, \bar{Q}(\chi, z, w)) \equiv w
$$

here and in what follows, we use the following notation: if $u(x)$ is an analytic function or formal power series in some set of variables $x$, then $\bar{u}(x)$ is the function or power series given by $\bar{u}(x):=\overline{u(\bar{x})}$.

A submanifold $M$ is said to be of finite type (in the sense of Kohn and BloomGraham) at $p$ if the (complex) Lie algebra $\mathfrak{g}_{M}$ generated by all smooth CR (or $(0,1)$ ) vector fields on $M$ and their conjugates satisfies $\mathfrak{g}_{M}(p)=\mathbb{C} T_{p} M$. Our proof of Theorem 1.1 will rely partly on a characterization of finite type due to Baouendi, Rothschild and the first author given in [BER96] (see also [BER03]) in terms of the generic rank of the iterated Segre mappings; precise details will be given in Section 3. A generic, real-analytic submanifold $M$ is said to be holomorphically nondegenerate at $p$ if there is no (nontrivial) germ at $p$ of a holomorphic vector field tangent to $M$ near $p$. By a germ at $p$ of a holomorphic vector field we shall mean a vector field of the form

$$
L=\sum_{j=1}^{N} \varphi_{j}(Z) \frac{\partial}{\partial Z_{j}}
$$

where the $\varphi_{j}$ are germs at $p$ of holomorphic functions.

We shall consider the variables $\bar{z}, \bar{w}$ in (2) as an independent set of complex variables $\xi=(\chi, \tau)$, and thus the complexified equation $w=Q(z, \chi, \tau)$ defines a complex submanifold $\mathcal{M}$ of codimension $d$ in $\mathbb{C}_{Z}^{N} \times \mathbb{C}_{\xi}^{N}$. We shall refer to $\mathcal{M}$ as the complexification of $M$. If $H(Z)$ is a holomorphic mapping $\left(\mathbb{C}^{N}, 0\right) \rightarrow\left(\mathbb{C}^{N}, 0\right)$ sending $M$ into $M^{\prime}$, then there is a $d \times d$ matrix $a(Z, \xi)$ of holomorphic functions in a neighborhood of the origin in $\mathbb{C}_{Z}^{N} \times \mathbb{C}_{\xi}^{N}$ such that the following identity holds:

$$
\rho^{\prime}(H(Z), \bar{H}(\xi))=a(Z, \xi) \cdot \rho(Z, \xi) .
$$

Equivalently, the complexified mapping $\mathcal{H}(Z, \xi):=(H(Z), \bar{H}(\xi))$ sends $\mathcal{M}$ into $\mathcal{M}^{\prime}$ (with the obvious notation). It is straightforward to verify that $H$ is $\mathrm{CR}$ transversal to $M^{\prime}$ at 0 if and only if $\operatorname{det} a(0) \neq 0$ (cf. [BER07]).

It is convenient to relax the convergence properties of the map and defining equations. A formal, generic submanifold $M$ of codimension $d$ through 0 in $\mathbb{C}^{N}$ is defined by a formal equation of the form (2), where $Q(z, \chi, \tau)$ is a $\mathbb{C}^{d}$-valued power series in $(z, \chi, \tau) \in \mathbb{C}^{n} \times \mathbb{C}^{n} \times \mathbb{C}^{d}$ satisfying the normality condition (3) and the reality condition (4). A formal holomorphic mapping $H:\left(\mathbb{C}^{N}, 0\right) \rightarrow\left(\mathbb{C}^{N}, 0\right)$ (i.e. a $\mathbb{C}^{N}$-valued power series in $Z=(z, w)$ with no constant term) is said to send the formal submanifold $M$ into a formal submanifold $M^{\prime}$ if there exists a $d \times d$ matrix $a(Z, \xi)$ of formal power series such that (5) holds. If $M$ and $M^{\prime}$ are smooth, generic submanifolds through $p$ and $p^{\prime}$ in $\mathbb{C}^{N}$ and $H$ is a holomorphic 
mapping $\left(\mathbb{C}^{N}, p\right) \rightarrow\left(\mathbb{C}^{N}, p^{\prime}\right)$ (or a smooth $\mathrm{CR}$ mapping defined on $M$ ) sending $M$ into $M^{\prime}$, then one can associate to $M$ and $M^{\prime}$ formal manifolds, still denoted by $M$ and $M^{\prime}$, through 0 and a formal holomorphic mapping, also denoted by $H:\left(\mathbb{C}^{N}, 0\right) \rightarrow\left(\mathbb{C}^{N}, 0\right)$, sending $M$ into $M^{\prime}$; the reader is e.g. referred to BER99b] for this (fairly obvious) construction. It is also straightforward to verify that the holomorphic mapping $H$ sending the smooth manifold $M$ into the smooth manifold $M^{\prime}$ is CR transversal to $M^{\prime}$ at $p$ if and only if the formal manifolds and mapping satisfy (5) with $\operatorname{det} a(0) \neq 0$. Thus, to prove Theorem 1.1 it suffices to consider formal manifolds $M$ and $M^{\prime}$ through 0 in $\mathbb{C}^{N}$, a formal holomorphic mapping $H:\left(\mathbb{C}^{N}, 0\right) \rightarrow\left(\mathbb{C}^{N}, 0\right)$ sending $M$ into $M^{\prime}$, i.e. satisfying (5), and prove that the matrix $a(Z, \xi)$ in (5) satisfies $\operatorname{det} a(0) \neq 0$; the reader is also referred to [ER06] for more details on this reduction to the formal case. In what follows, we shall consider only formal mappings and formal generic submanifolds.

\section{Proof of the main theorem And Corollaries}

In this section, $M$ and $M^{\prime}$ are generic formal submanifolds through 0 , given in normal coordinates by $\rho(Z, \bar{Z})=0$ and $\rho^{\prime}\left(Z^{\prime}, \bar{Z}^{\prime}\right)=0$, where $\rho(Z, \xi)=w-Q(z, \chi, \tau)$ and $\rho^{\prime}\left(Z^{\prime}, \xi^{\prime}\right)=w^{\prime}-Q^{\prime}\left(z^{\prime}, \chi^{\prime}, \tau^{\prime}\right)$, and $H(Z)$ is a formal mapping sending $M$ into $M^{\prime}$. For the proof of the main result, we shall need a sequence of lemmas. Let us begin with the following lemma.

Lemma 3.1. Let $M$ and $M^{\prime}$ be generic formal submanifolds defined as above and $H:\left(\mathbb{C}^{N}, 0\right) \rightarrow\left(\mathbb{C}^{N}, 0\right)$ a formal mapping sending $M$ into $M^{\prime}$, that is,

$$
\rho^{\prime}(H(Z), \bar{H}(\xi))=a(Z, \xi) \cdot \rho(Z, \xi),
$$

where $a(Z, \xi)$ is a $d \times d$ matrix of formal power series in $\mathbb{C}[[Z, \xi]]$. Then, there are $d \times d$ matrices $C(Z, \xi)$ and $E(Z, \xi)$ of formal power series in $\mathbb{C}[[Z, \xi]]$ such that the following holds:

$$
\begin{aligned}
\operatorname{det} H_{Z}(Z) \cdot I_{d} & =a(Z,(\chi, \bar{Q}(\chi, Z))) \cdot C(Z, \xi), \\
\operatorname{det} \bar{H}_{\xi}(\xi) \cdot I_{d} & =a((z, Q(z, \xi)), \xi) \cdot E(z, \xi),
\end{aligned}
$$

where $I_{d}$ denotes the $d \times d$ identity matrix.

Proof. By differentiating (6) with respect to $Z$ we get

$$
\rho_{Z^{\prime}}^{\prime}(H(Z), \bar{H}(\xi)) \cdot H_{Z}(Z)=a_{Z}(Z, \xi) \cdot \rho(Z, \xi)+a(Z, \xi) \cdot \rho_{Z}(Z, \xi) .
$$

Now we substitute $\xi=(\chi, \bar{Q}(\chi, Z))$ into (9) and note that $\rho(Z,(\chi, \bar{Q}(\chi, Z))))=0$. Hence

(10) $\rho_{Z^{\prime}}^{\prime}(H(Z), \bar{H}(\chi, \bar{Q}(\chi, Z))) \cdot H_{Z}(Z)=a(Z,(\chi, \bar{Q}(\chi, Z))) \cdot \rho_{Z}(Z,(\chi, \bar{Q}(\chi, Z)))$.

By Cramer's rule, there is an $N \times N$ matrix of formal power series $B(Z)$ such that $H_{Z} B=B H_{Z}=\left(\operatorname{det} H_{Z}\right) I_{N}$. Thus, it follows from equation (10) that

$$
\begin{aligned}
& \rho_{Z^{\prime}}^{\prime}(H(Z), \bar{H}(\chi, \bar{Q}(\chi, Z))) \cdot \operatorname{det} H_{Z}(Z) \\
& \quad=a(Z,(\chi, \bar{Q}(\chi, Z))) \cdot \rho_{Z}(Z,(\chi, \bar{Q}(\chi, Z))) \cdot B(Z) .
\end{aligned}
$$


Recall that $\rho^{\prime}\left(Z^{\prime}, \xi^{\prime}\right)=w^{\prime}-Q\left(z^{\prime}, \xi^{\prime}\right)$, where $Z^{\prime}=\left(z^{\prime}, w^{\prime}\right)$. It then follows that the last $d$ columms of $\rho_{Z^{\prime}}^{\prime}$ is the identity matrix $I_{d}$. Thus, by considering the last $d$ columns of equation (11), we find that there is a $d \times d$ matrix $C=C(Z, \xi)$ whose entries are power series such that

$$
\operatorname{det} H_{Z}(Z) \cdot I_{d}=a(Z,(\chi, \bar{Q}(\chi, Z))) \cdot C(Z, \xi) .
$$

Thus, equation (7) is proved.

Now, to prove (8), we differentiate (6) with respect to $\xi$ and substitute $Z=$ $(z, Q(z, \xi))$. Since $\rho((z, Q(z, \xi)), \xi)=0$ we have

$$
\rho_{\xi^{\prime}}^{\prime}(H(z, Q(z, \xi)), \bar{H}(\xi)) \cdot \bar{H}_{\xi}(\xi)=a((z, Q(z, \xi)), \xi) \cdot \rho_{\xi}((z, Q(z, \xi)), \xi) .
$$

Note that $\bar{H}_{\xi}(\xi) \cdot \bar{B}(\xi)=\operatorname{det} \bar{H}_{\xi}(\xi) I_{N}$, where $B(Z)$ is the matrix introduced above. Multiplying both sides of (12) with $\bar{B}(\xi)$, we obtain

$$
\rho_{\xi^{\prime}}^{\prime}(H(z, Q(z, \xi)), \bar{H}(\xi)) \cdot \operatorname{det} \bar{H}_{\xi}(\xi)=a((z, Q(z, \xi)), \xi) \cdot \rho_{\xi}((z, Q(z, \xi)), \xi) \cdot \bar{B}(\xi) .
$$

Taking the last $d$ columns of (13), we obtain

$$
\operatorname{det} \bar{H}_{\xi}(\xi) \cdot \rho_{\tau^{\prime}}^{\prime}(H(z, Q(z, \xi)), \bar{H}(\xi))=a((z, Q(z, \xi)), \xi) \cdot D(Z, \xi),
$$

where $D(Z, \xi)$ is the matrix formed by the last $d$ columns of $\rho_{\xi}((z, Q(z, \xi)), \xi)$. $\bar{B}(\xi)$. Now, it follows from (3) that $\rho_{\tau^{\prime}}^{\prime}(0,0)=Q_{\tau^{\prime}}^{\prime}(0,0,0)=I_{d}$ and hence $\left.\rho_{\tau^{\prime}}^{\prime}(H(z, Q(z, \xi)), \bar{H}(\xi))\right)$ is invertible over the ring $\mathbb{C}[[Z, \xi]]$. Consequently, it follows from (14) that there is a matrix $E(Z, \xi)$ such that

$$
\operatorname{det} \bar{H}_{\xi}(\xi) \cdot I_{d}=a((z, Q(z, \xi)), \xi) \cdot E(Z, \xi),
$$

which is (8). The proof is complete.

Lemma 3.2. Assume that $\operatorname{det} H_{Z}(0)=0$, but $\operatorname{det} H_{Z}(Z) \not \equiv 0$. Then there exist units $u(Z, \xi), v(Z, \xi)$ in $\mathbb{C}[[Z, \xi]]$ and formal power series $b(Z) \in \mathbb{C}[[Z]], c(\xi) \in \mathbb{C}[[\xi]]$ such that

$$
\begin{aligned}
\operatorname{det} a(Z,(\chi, \bar{Q}(\chi, Z))) & =u(Z, \xi) \cdot b(Z) ; \\
\operatorname{det} a((z, Q(z, \xi)), \xi) & =v(Z, \xi) \cdot c(\xi) .
\end{aligned}
$$

Furthermore, $b(Z)$ is a divisor of $\left(\operatorname{det} H_{Z}(Z)\right)^{d}$ in $\mathbb{C}[[Z]]$ and $c(\xi)$ is a divisor of $\left(\operatorname{det} \bar{H}_{\xi}(\xi)\right)^{d}$ in the ring $\mathbb{C}[[\xi]]$.

Proof. It follows from (7) that

$$
\left(\operatorname{det} H_{Z}(Z)\right)^{d}=\operatorname{det} a(Z,(\chi, \bar{Q}(\chi, Z))) \operatorname{det} C(Z,(\chi, \bar{Q}(\chi, Z))) .
$$

We now factor both sides of (17) into products of irreducible elements in the unique factorization domain $\mathbb{C}[[Z, \xi]]$. Since the left-hand side of (17) is a nontrivial formal power series in the ring $\mathbb{C}[[Z]] \subset \mathbb{C}[[Z, \xi]]$, its factorization involves factors that are power series in $Z$ only. Thus, by the uniqueness of the factorization, we obtain

$$
\operatorname{det} a(Z,(\chi, \bar{Q}(\chi, Z)))=u(Z, \xi) \cdot b(Z),
$$

where $b(Z) \in \mathbb{C}[[Z]]$ is a divisor of $\left(\operatorname{det} H_{Z}(Z)\right)^{d}$ and $u(Z, \xi)$ is a unit in $\mathbb{C}[[Z, \xi]]$.

Similarly, it follows from (8) that

$$
\left(\operatorname{det} \bar{H}_{\xi}(\xi)\right)^{d}=\operatorname{det} a((z, Q(z, \xi)), \xi) \cdot \operatorname{det} E(Z, \xi) .
$$


A similar argument to the one above shows that

$$
\operatorname{det} a((z, Q(z, \xi)), \xi)=v(Z, \xi) \cdot c(\xi),
$$

where $v(Z, \xi) \in \mathbb{C}[[Z, \xi]]$ is a unit and $c(\xi) \in \mathbb{C}[[\xi]]$ is a divisor of $\left(\operatorname{det} \bar{H}_{\xi}(\xi)\right)^{d}$. The proof is complete.

Lemma 3.3. Let $u, v, b, c$ be as in Lemma 3.2. Then, there is a unit $s(Z, \xi)$ in $\mathbb{C}[[Z, \xi]]$ such that

$$
b(z, Q(z, \xi))=s(Z, \xi) \cdot c(\xi) .
$$

Proof. We substitute $Z=(z, Q(z, \xi))$ into (15), and use (4) and (16) to obtain

$$
\begin{aligned}
u((z, Q(z, \xi)), \xi) \cdot b(z, Q(z, \xi)) & =\operatorname{det} a((z, Q(z, \xi)),(\chi, \bar{Q}(\chi, z, Q(z, \xi)))) \\
& =\operatorname{det} a((z, Q(z, \xi)), \xi) \\
& =v(Z, \xi) \cdot c(\xi) .
\end{aligned}
$$

Since $u(Z, \xi)$ and $v(Z, \xi)$ are units, we can take $s(Z, \xi)=(u((z, Q(z, \xi)), \xi))^{-1} v(Z, \xi)$ to obtain (18). It is obvious that $s(Z, \xi)$ is also a unit. The proof is complete.

Let $\phi:\left(\mathbb{C}^{m}, 0\right) \rightarrow\left(\mathbb{C}^{N}, 0\right)$ be a formal mapping. Thus, $\phi=\left(\phi_{1}(x), \phi_{2}(x), \ldots\right.$, $\phi_{N}(x)$ ), where $\phi_{j}(x)$ are formal power series in $x$ with no constant term. Recall that the generic rank of $\phi$ is the rank of the Jacobi matrix $\left(\partial \phi_{j} / \partial x_{k}\right)$ considered as a matrix with entries in the quotient field of $\mathbb{C}[[x]]$. We will need the following lemma, whose proof for the case $k=1$ and $m=N$ may be found, e.g., in BER99a, Proposition 5.3.5. The same proof works also in the general case, with some obvious modifications.

Lemma 3.4. Let $K:\left(\mathbb{C}^{N}, 0\right) \rightarrow\left(\mathbb{C}^{k}, 0\right)$ and $\phi:\left(\mathbb{C}^{m}, 0\right) \rightarrow\left(\mathbb{C}^{N}, 0\right)$ be formal mappings. Assume that $\phi$ has generic rank $N$. If $K \circ \phi \equiv 0$, then $K \equiv 0$.

We shall now prove our main result.

Proof of Theorem 1.1. As explained in Section 2, we may assume that $M$ and $M^{\prime}$ are formal manifolds through $0 \in \mathbb{C}^{N}, H:\left(\mathbb{C}^{N}, 0\right) \rightarrow\left(\mathbb{C}^{N}, 0\right)$ a formal mapping satisfying (5D), and then to prove Theorem 1.1, it suffices to prove that the matrix $a(Z, \xi)$ in (5) satisfies $\operatorname{det} a(0) \neq 0$. Thus, we assume, in order to reach a contradiction, that $\operatorname{det} a(0)=0$. We deduce from (16) that

$$
v(0,0) \cdot c(0)=\operatorname{det} a(0)=0 .
$$

Thus, $c(0)=0$ since $v(Z, \xi)$ is a unit. Setting $\xi=0$ in equation (18) yields

$$
b(z, 0)=b(z, Q(z, 0))=s((z, 0), 0) \cdot c(0)=0 .
$$

Thus, it follows from (15) that

$$
\operatorname{det} a(z, 0, \chi, \bar{Q}(\chi, z, 0))=u(z, 0, \chi, \bar{Q}(\chi, z, 0)) \cdot b(z, 0)=0 .
$$

By taking determinants on both sides of equation (8), substituting $\xi=(\chi, \bar{Q}(\chi, z, 0))$ and using (4), we conclude that

$$
\begin{aligned}
& \left(\operatorname{det} \bar{H}_{\xi}(\chi, \bar{Q}(\chi, z, 0))\right)^{d} \\
& \quad=\operatorname{det} a(z, 0, \chi, \bar{Q}(\chi, z, 0)) \cdot \operatorname{det} E(Z,(\chi, \bar{Q}(\chi, z, 0))) \equiv 0 .
\end{aligned}
$$

In the hypersurfaces case, the proof is complete. Indeed, if $M$ is of finite type at 0 , then the map $(z, \chi) \mapsto(\chi, \bar{Q}(\chi, z, 0))$ has rank $n+1$ (see e.g [BER96]) and thus, by Lemma 3.4. equation (21) implies that $\operatorname{det} \bar{H}_{\xi} \equiv 0$. This is a contradiction. 
For the general case, we will need the iterated Segre mappings introduced in BER96 (see also BER03, ER06]). For a positive integer $k$, the $k$ th Segre mapping of $M$ at 0 is the mapping $v^{k}: \mathbb{C}^{k n} \rightarrow \mathbb{C}^{N}$ defined by

$$
\mathbb{C}^{k n} \ni t=\left(t^{1}, \ldots, t^{k}\right) \mapsto v^{k}(t):=\left(t^{k}, u^{k}\left(t^{1}, \ldots, t^{k}\right)\right),
$$

where $u^{k}: \mathbb{C}^{k n} \rightarrow \mathbb{C}^{d}$ is given inductively by

$$
u^{1}\left(t^{1}\right)=0, u^{k}\left(t^{1}, \ldots, t^{k}\right)=Q\left(t^{k}, t^{k-1}, \overline{u^{k-1}}\left(t^{1}, \ldots, t^{k-1}\right)\right), k \geq 2 .
$$

The crucial property of the Segre mappings needed here is the result (see BER96, BER99a, BER99b, BER03]) that $M$ is of finite type at 0 if and only if the maps $v^{k}$ have generic rank $N$ for $k$ large enough ( $k \geq d+1$ suffices). Thus, by Lemma 3.4, the following lemma implies that $\operatorname{det} H_{Z} \equiv 0$, which is a contradiction and completes the proof of Theorem 1.1 .

Lemma 3.5. For every $j \geq 0$, the following holds:

$$
\operatorname{det} H_{Z} \circ v^{2 j+1} \equiv 0 .
$$

Proof. We may consider $b(Z)$ and $c(\xi)$ in Lemmas 3.2 and 3.3 as power series in $(Z, \xi)$ by $b(Z, \xi)=b(Z)$ and $c(Z, \xi)=c(\xi)$. Since the complexification $\mathcal{M}$ of $M$ is parametrized by

$$
(z, \chi, \tau) \mapsto(z, Q(z, \chi, \tau), \chi, \tau)
$$

it follows from (18) that $b \cong c$ on $\mathcal{M}$, where we use the notation $\alpha \cong \beta$ to mean $\alpha=\gamma \beta$ for some unit $\gamma$. Now, another crucial property of the Segre mappings $v^{k}$ (see e.g. [BER99b]) is that $\left(v^{k+1}, \overline{v^{k}}\right) \in \mathcal{M}$ and $\left(v^{k-1}, \overline{v^{k}}\right) \in \mathcal{M}$ for every $k$. Consequently, equation (18) implies that

$$
b \circ v^{k+1} \cong c \circ \overline{v^{k}}, \quad c \circ \overline{v^{k}} \cong b \circ v^{k-1} .
$$

We deduce that $b \circ v^{k+1} \cong b \circ v^{k-1}$ for all $k \geq 2$. By induction, we obtain, for every positive integer $j$,

$$
b \circ v^{2 j+1} \cong b \circ v^{1} .
$$

Hence, since $b \circ v^{1} \equiv 0$ by (19), we conclude that $b \circ v^{2 j+1} \equiv 0$. Since, by Lemma 3.2, $b(Z)$ is a divisor of $\left(\operatorname{det} H_{Z}(Z)\right)^{d}$, it follows that

$$
\operatorname{det} H_{Z} \circ v^{2 j+1} \equiv 0 \text {. }
$$

This completes the proof of Lemma 3.5 .

We may now prove Theorem 1.4

Proof of Theorem 1.4. Since Jac $H \not \equiv 0$ and $M$ is of finite type at $p$, it follows from Proposition 2.3 in [ER06] that $M^{\prime}$ is of finite type at $p^{\prime}$. Also, by Theorem 1.1, $H$ is CR transversal to $M^{\prime}$ at $p$ and thus, in particular, transversally regular to $M^{\prime}$ (see [ER06]). Consequently, by Theorem 6.1 in ER06, if $M$ is also essentially finite at $p$, then $H$ is a finite mapping and $M^{\prime}$ is essentially finite at $p^{\prime}$. If, in addition, $M$ is finitely nondegenerate at p, then Theorem 6.6 in [ER06] asserts that $H$ is a local biholomorphism near $p$.

Now, to prove Theorem 1.3, we shall need the following proposition. A different proof of a slightly more general result in the hypersurface case may be found in LM06, (see Theorem 2.8). 
Proposition 3.6. Let $M, M^{\prime} \subset \mathbb{C}^{N}$ be smooth generic submanifolds of the same dimension through 0 and $H:\left(\mathbb{C}^{N}, 0\right) \rightarrow\left(\mathbb{C}^{N}, 0\right)$ a germ of a holomorphic mapping such that $H(M) \subset M^{\prime}$. Assume that $M$ is holomorphically nondegenerate at 0 . If $H$ is $C R$ transversal to $M^{\prime}$ at 0 , then $\operatorname{Jac} H \not \equiv 0$.

Proof. The idea of the proof is taken from [A08]. Assume, in order to reach a contradiction, that Jac $H \equiv 0$. Then, there is a nontrivial $N$-vector $U(Z)$ with components in the field of fractions of $\mathbb{C}[[Z]]$ such that

$$
H_{Z}(Z) \cdot U(Z) \equiv 0
$$

By multiplying (26) with a suitable power series if necessary, we may assume that $U(Z)$ has components in $\mathbb{C}[[Z]]$. Thus, we can consider the following nontrivial formal holomorphic vector field:

$$
L=\sum_{j=1}^{n} U_{j}(Z) \frac{\partial}{\partial Z_{j}}
$$

It follows from (26) that $L H_{j}=0$ for all $j=1, \ldots, N$. Now, since $H$ sends $M$ into $M^{\prime}$ we have

$$
\rho^{\prime}(H(Z), \bar{H}(\xi))=a(Z, \xi) \cdot \rho(Z, \xi) .
$$

Applying $L$ to the left-hand side of (27), we obtain

$$
\sum_{j=1}^{N} \rho_{Z_{j}^{\prime}}^{\prime}(H(Z), \bar{H}(\xi)) L H_{j}(Z) \equiv 0 .
$$

Consequently, we must also have $L(a(Z, \xi) \cdot \rho(Z, \xi)) \equiv 0$. In other words,

$$
(L a) \cdot \rho+a \cdot(L \rho) \equiv 0 .
$$

Since $H$ is CR transversal to $M^{\prime}$ at 0 , we have $\operatorname{det} a(0) \neq 0$ and hence $a(Z, \xi)$ is invertible in $\mathbb{C}[[Z, \xi]]$. We deduce from (28) that

$$
L \rho=-(a)^{-1}(L a) \rho .
$$

It follows that $L$ is tangent to $M$. This is a contradiction since $M$ is holomorphically nondegenerate. The proof is complete.

Theorem 1.3 is now a direct consequence of Theorem 1.1 and Proposition 3.6 .

\section{Acknowledgement}

The authors would like to thank the anonymous referee for useful comments, especially for pointing out Example 1.2

\section{REFERENCES}

[A08] Angle, R., Geometric properties and related results for holomorphic Segre preserving maps. arXiv:0810.2570v1

[CR94] Chirka, E. M. and Rea, C., Normal and tangent ranks of CR mappings. Duke Math. $J .$, 76, 417-431, (1994). MR1302319 (95j:32041)

[CR98] Chirka, E. M. and Rea, C., Differentiable CR mappings and CR orbits. Duke Math. J., 94, 325-340 (1998). MR1638591 (99f:32015)

[BER96] Baouendi, M. S., Ebenfelt, P., and Rothschild, L. P., Algebraic properties of holomorphic mappings between real algebraic sets in $\mathbb{C}^{n}$. Acta Math., 177, 225-273 (1996). MR:1440933 (99b:32030) 
[BER98] Baouendi, M. S., Ebenfelt, P., and Rothschild, L. P., CR automorphisms of real analytic manifolds in complex space. Comm. Anal. Geom., 6, 291-315 (1998). MR.1651418 (99i:32024)

[BER99a] Baouendi, M. S., Ebenfelt, P., and Rothschild, L. P., Real Submanifolds in Complex Space and Their Mappings. Princeton Math. Series 47, Princeton Univ. Press, 1999. MR 1668103 (2000b:32066)

[BER99b] Baouendi, M. S., Ebenfelt. P., and Rothschild, L. P., Rational dependence of smooth and analytic CR mappings on their jets. Math. Ann. 315, 205-249 (1999). MR.1721797 (2001b:32075)

[BER03] Baouendi, M. S., Ebenfelt. P., and Rothschild, L. P., Dynamics of the Segre varieties of a real submanifold in complex space. J. Algebraic Geom. 12, 81-106 (2003). MR1948686 (2004f:32050)

[BER07] Baouendi, M. S., Ebenfelt. P., and Rothschild, L. P., Transversality of holomorphic mappings between real hypersurfaces in different dimensions. Comm. Anal. Geom., 15, 589-611 (2007). MR2379806 (2009a:32019)

[BHR95] Baouendi, M. S., Huang, X., and Rothschild, L. P., Nonvanishing of the differential of holomorphic mappings at boundary points. Math. Res. Lett., 2, 737-750 (1995). MR:1362966 (97d:32025)

[BR90] Baouendi, M. S. and Rothschild, L. P., Geometric properties of mappings between hypersurfaces in complex space. J. Differential Geom., 31, 473-499 (1990). MR.1037411 (91g:32032)

[BR93] Baouendi, M. S. and Rothschild, L. P., A generalized complex Hopf lemma and its applications to CR mappings. Invent. Math., 111, 331-348 (1993). MR.1198813 (95c:32017)

[BB82] Bedford, E. and Bell, S., Proper self-maps of weakly pseudoconvex domains. Math. Ann., 261, 47-49 (1982). MR675205 (84c:32026)

[ER06] Ebenfelt, P. and Rothschild, L. P., Transversality of CR mappings. Amer. J. Math., 128, 1313-1343 (2006). MR2262176 (2008b:32024)

[Forn76] Fornaess, J. E., Embedding strictly pseudoconvex domains in convex domains. Amer. J. Math., 98, 529-569 (1976). MR0422683 (54:10669)

[Forn78] Fornaess, J. E., Biholomorphic mappings between weakly pseudoconvex domains. $P a$ cific J. Math., 74, 63-65 (1978). MR0481111 (58:1257)

[GT83] Gilbarg, D. and Trudinger, N. S., Elliptic partial differential equations of second order. Second edition. Grundlehren der Mathematischen Wissenschaften 224. SpringerVerlag, Berlin, 1983. MR.737190 (86c:35035)

[HP96] Huang, X. and Pan, Y., Proper holomorphic mappings between real analytic domains in $C^{n}$. Duke Math. J., 82, 437-446 (1996). MR1387236 (97f:32036)

[LM06] Lamel, B. and Mir, N., Remarks on the rank properties of formal CR maps. Science in China Series A: Mathematics, 49 (11), 1477-1490 (2006). MR2288209(2007k:32049)

[P77] Pinčuk, S. I., Analytic continuation of mappings along strictly pseudo-convex hypersurfaces. (Russian) Dokl. Akad. Nauk SSSR, 236, 544-547 (1977). MR0481115(58:1261)

Department of Mathematics, University of California at San Diego, La Jolla, CaliFORNIA 92093-0112

E-mail address: pebenfel@math.ucsd.edu

Department of Mathematics, University of California at San Diego, La Jolla, CaliFORNIA 92093-0112

E-mail address: snduong@math.ucsd.edu 